\title{
Efficient method of assembly sequence planning based on GAAA and optimizing by assembly path feedback for complex product
}

\author{
Cheng Hui • Li Yuan • Zhang Kai-fu
}

Published online: 15 October 2008

(C) Springer-Verlag London Limited 2008

\section{Erratum to: Int J Adv Manuf Technol}

DOI 10.1007/s00170-008-1661-8

Unfortunately, an error occurred in Acknowledgement.

The correct Acknowledgement is given below.

We gratefully acknowledge the support of the Nation High-Tech. R\&D Program of China (863) under grant 2006AA04Z137, the Doctorate Foundation of Northwestern Polytechnical University (CX200809) and Aoxiang Star Program in Northwestern Polytechnical University, China. We would also like to thank the editors and the anonymous referees for their insightful comments.

\footnotetext{
The online version of the original article can be found at http://dx.doi. org/10.1007/s00170-008-1661-8.

C. Hui $(\bowtie) \cdot$ L. Yuan $\cdot$ Z. Kai-fu

The Ministry of Education Key Lab of Contemporary,

Design and Integrated Manufacturing Technical,

Northwestern Polytechnical University,

Xi'an, China

e-mail: chenghui_mercury@sina.com

L. Yuan

e-mail: yuanli@nwpu.edu.cn

Z. Kai-fu

e-mail: zhangkf@nwpu.edu.cn
} 